\title{
Medición del capital estructural de la organización: una investigación en el contexto de la Universidad Autónoma de Manizales*
}

\author{
Measurement of the Organizational Structural \\ Capital: A Research in the Context of the Univer- \\ sidad Autónoma de Manizales
}

\section{Medição do capital estrutural da organização: uma pesquisa no contexto da Universidad Autónoma de Manizales}

\section{Cristhian Guillermo Naranjo Herrera ${ }^{\star \star}$ Mónica Andrea Chu Salgado ${ }^{* *}$}

Fecha de recibido: 27 de mayo de 2014

Fecha de aprobado: 6 de noviembre de 2014

Doi: dx.doi.org/10.12804/rev.univ.empresa.29.2015.05

Para citar este artículo: Naranjo Herrera, C. G., \& Chu Salgado, M. A. (2015). Medición del capital estructural de la organización: una investigación en el contexto de la Universidad Autónoma de Manizales. Universidad \& Empresa, 17(29), 11 1-130. Doi: dx.doi.org/10.12804/rev.univ.empresa.29.2015.05

Este artículo es resultado del proyecto de investigación titulado "El capital intelectual en la Universidad Autónoma de Manizales”, desarrollado en el contexto de la Maestría en Administración de Negocios de la Universidad Autónoma de Manizales, Colombia.

** Doctor en Administración de Empresas y Dirección de Recursos Humanos de la Universidad de Málaga (España), Magíster en Administración del Instituto Tecnológico de Estudios Superiores de Monterrey (México) y la Universidad Nacional Autónoma de Bucaramanga (Colombia), Especialista en Administración de Recursos Humanos de la Universidad de Manizales (Manizales, Colombia), Abogado de la Universidad de Caldas (Manizales, Colombia). Profesor Asociado del Departamento de Administración de la Universidad Autónoma de Manizales (Colombia). Investigador principal. Correo electrónico: cnaranjo@autonoma.edu.co

**** Magíster en Administración de Negocios de la Universidad Autónoma de Manizales (Colombia), Especialista en Desarrollo Gerencial de la Universidad Autónoma de Manizales (Colombia), Ingeniera de Sistemas y Telecomunicaciones de la Universidad Autónoma de Manizales (Manizales, Colombia). Coinvestigadora. Correo electrónico: mchu@autonoma.edu.co 


\section{RESUMEN}

Esta investigación se desarrolló con base en dos objetivos: 1) generar un instrumento mediante el cual las organizaciones puedan medir su capital estructural y 2) proporcionar evidencia empírica de este, bajo el marco conceptual del capital intelectual. Se realizó un estudio cuantitativo y descriptivo, de corte empírico-analítico. Mediante este se identificó, midió y analizó el capital estructural de la Universidad Autónoma de Manizales (UAM) para los años 2011 y 2012. Los resultados ponen en evidencia que es posible medir el capital estructural en una organización y, de esta manera, comprender su dinámica. Esto puede contribuir a la optimización de la gestión de sus activos intangibles. En la UAM, específicamente, el capital estructural constituye una fortaleza. Esta se evidencia en la constante inversión en infraestructura tecnológica y su continuo fortalecimiento en el campo de la investigación, el desarrollo y la innovación ( $\mathrm{I}+\mathrm{D}+\mathrm{i})$.

Palabras clave: Capital estructural, capital intelectual, medición del capital estructural.

\section{ABSTRACT}

This research had two objectives: 1) to provide an instrument with which organizations could measure their structural capital and 2) to offer the respective empirical evidence within the conceptual framework of intellectual capital. An empirical-analytical quantitative and descriptive study was undertaken. Using this study, the structural capital of the Universidad Autonoma de Manizales (UAM) in 2011 and 2012 was identified, measured and analyzed. The results showed that it is possible to measure structural capital in an organization and thus understand its dynamics. This can contribute to optimizing the management of its intangible assets. Specifically, in the case of UAM, its structural capital is a strength. This is evidenced in its constant investment in technological infrastructure and its continuous strengthening in the area of research, development and innovation (R\&D+i).

Keywords: Structural capital, intellectual capital, measurement of structural capital.

\section{RESUMO}

Esta pesquisa de desenvolvimento com base em dois objetivos: 1) gerar um instrumento mediante o qual as organizações possam medir seu capital estrutural e 2) proporcionar evidência empírica deste, sob o marco conceitual do capital intelectual. Realizou-se um estudo quantitativo e descritivo, de corte empírico-analítico. Mediante este se identificou, mediu e analisou o capital estrutural da Universidad Autónoma de Manizales (UAM) para os anos 2011 e 2012. Os resultados põem em evidência que é possível medir o capital estrutural em uma organização e, desta maneira, compreender a sua dinâmica. Isto pode contribuir à optimização da gestão de seus ativos intangíveis. Na UAM, especificamente, o capital estrutural constitui uma fortaleza. Esta se evidencia no constante investimento em infraestrutura tecnológica e seu contínuo fortalecimento no campo da Investigação, Desenvolvimento e Inovação (I+D+i).

Palavras-chave: capital estrutural, capital intelectual, medição do capital estrutural. 


\section{INTRODUCCIÓN Y REVISIÓN DE LA LITERATURA}

La era actual está caracterizada por la demanda de nuevas formas de gestionar el valor en la economía a partir de los recursos intangibles y de los activos basados en conocimiento (Klein, 1998; Marr, Gupta, Pike, \& Roos, 2003). En este contexto, el capital humano ocupa un lugar cada vez más relevante. Los aspectos intangibles vinculados con las organizaciones, en particular, no son un asunto trivial. Estos tienen una importancia creciente en la estructura de valor de la gran mayoría de los bienes y servicios.

La competitividad de las organizaciones, que se sustenta cada vez de manera amplia en uso de los activos intangibles (Low \& Cohen, 2004), puede entenderse como la capacidad de identificar y aprovechar, de forma permanente y sostenible, en un escenario específico, las ventajas económicas propias de un mercado global. Esta tiene que ver con la posición relativa que ocupa una organización en relación con sus competidores, así como con la aptitud y capacidad para sostenerla y mejorarla a través del tiempo. Para ello, en efecto, resulta esencial contar con un adecuado capital intangible (Bueno Campos, 1998).

Dicha situación no ha pasado desapercibida en los mercados. Estos, desde la década de los noventa hasta nuestros días, han ido poniendo cada vez más de manifiesto la diferencia significativa existente entre el valor que otorgan los actores a las empresas y el valor recogido en las cuentas anuales (Cañibano \& Sánchez, 2004). Esto de acuerdo con criterios valorativos y principios de contabilidad generalmente aceptados. Sin embargo, el auge reciente de estas consideraciones, y el grupo de definiciones desarrolladas respecto al tema (ver tabla 1), no debe hacer olvidar que, desde épocas anteriores, existían voces autorizadas que llamaban la atención acerca de la importancia de los recursos intangibles.

Tabla 1. Definiciones de capital intelectual

\begin{tabular}{|l|l|}
\hline \multicolumn{1}{|c|}{ Autores } & \multicolumn{1}{c|}{ Definición } \\
\hline Bontis (1996) & $\begin{array}{l}\text { Diferencia entre el valor de mercado de la empresa y el costo de reposición } \\
\text { de sus activos. }\end{array}$ \\
\hline Brooking (1997) & Combinación de activos inmateriales que permite que la empresa funcione. \\
\hline $\begin{array}{l}\text { Bueno Campos } \\
(1998)\end{array}$ & $\begin{array}{l}\text { Conjunto de competencias básicas distintivas de carácter intangible que faci- } \\
\text { litan crear y sostener la ventaja competitiva. }\end{array}$ \\
\hline
\end{tabular}




\begin{tabular}{|l|l|}
\hline \multicolumn{1}{|c|}{ Autores } & \multicolumn{1}{c|}{ Definición } \\
\hline $\begin{array}{l}\text { Edvinsson \& } \\
\text { Malone (1997) }\end{array}$ & Conocimiento que puede convertirse en valor. \\
\hline Euroforum (1998) & $\begin{array}{l}\text { Conjunto de activos intangibles de una organización que, pese a no estar re- } \\
\text { flejados en los estados contables tradicionales, genera valor en la actualidad o } \\
\text { tiene potencial de generarlo en el futuro. }\end{array}$ \\
\hline Mantilla (2004) & $\begin{array}{l}\text { Conjunto de sistemas y procesos conformado por el capital humano, el capital } \\
\text { estructural y el capital relacional, orientados a la producción y participación } \\
\text { de conocimiento en función de objetivos estratégicos. }\end{array}$ \\
\hline $\begin{array}{l}\text { Mouritsen, Bukh, } \\
\text { Larsen \& Johansen } \\
\text { (2002) }\end{array}$ & $\begin{array}{l}\text { Fuentes de conocimiento para toda la organización que, en combinación, po- } \\
\text { seen capacidades y hacen posible que la organización pueda tomar medidas. }\end{array}$ \\
\hline $\begin{array}{l}\text { Nazari \& } \\
\text { Herremans (2007) }\end{array}$ & $\begin{array}{l}\text { Conjunto de elementos de naturaleza inmaterial que son imprescindibles para } \\
\text { que la empresa pueda llevar a cabo su actividad en un entorno basado en una } \\
\text { economía del conocimiento. }\end{array}$ \\
\hline Rastogi (2002) & $\begin{array}{l}\text { Capacidad integral de la empresa o capacidad-meta para afrontar los retos y } \\
\text { aprovechar las oportunidades en su interés continuo de buscar valor. }\end{array}$ \\
\hline Steward (1998) & $\begin{array}{l}\text { La suma de todos los conocimientos que poseen los empleados de una empre- } \\
\text { sa que le dan una ventaja competitiva. El material intelectual (conocimientos, } \\
\text { información, propiedad intelectual y experiencia) que se puede aprovechar } \\
\text { para crear riqueza. }\end{array}$ \\
\hline Swart (2006) & $\begin{array}{l}\text { El rendimiento tangible en forma de productos y servicios dentro del merca- } \\
\text { do de la empresa. El conocimiento único y valioso y las habilidades (tanto } \\
\text { individuales como colectivas) están integrados dentro de estos productos y } \\
\text { servicios. }\end{array}$ \\
\hline
\end{tabular}

Fuente: Elaboración propia.

El capital estructural, de algún modo, corresponde con la "perspectiva de los procesos internos" considerada dentro del discurso del cuadro de mando integral (Balanced Scorecard, BSC, Kaplan \& Norton, 1996). Este incluye aspectos como los procesos internos y de innovación. En planteamientos de otros autores, como Sveiby (1997), este se vincula con la idea de 'estructura interna', la cual tiene en cuenta la cultura, la organización informal, las redes internas y los sistemas informáticos y administrativos. Por su parte, en el contexto del modelo del navegador de Skandia (Edvinsson \& Malone, 1997), dicho capital está relacionado con el 'enfoque de procesos', que contempla los procesos organizativos y las tecnologías de la información.

Un importante grupo de autores (Bontis, 1998; Chen, Zhu, \& Yuan, 2004; Edvinsson \& Malone, 1997; 
Euroforum, 1998; Joia, 2004; McElroy, 2002; Ordóñez de Pablos, 2004a, 2004b; Saint Onge, 1996) denominan a este capital 'estructural' y establecen las diferentes dimensiones que lo componen. Entre los esfuerzos por realizar esta labor se encuentran los de Saint Onge (1996), quien identifica cuatro componentes, a saber: de sistemas, estructura, estrategia y cultura organizativa, y los de Edvinsson \& Malone (1997), para quienes se encuentra integrado por hardware, software, bases de datos, estructura, patentes, marcas y relaciones con los clientes, aspecto este último que, como se mostrará luego, es más propio del capital relacional.

Está también el planteamiento del Euroforum (1998), una entidad que sostiene que este capital está conformado por la cultura, la filosofía, la estructura, los procesos y la propiedad intelectual de la empresa. Para Bontis (1998), por su parte, son las rutinas organizativas, la estructura, la cultura y los sistemas de información los principales aspectos que le dan forma. De acuerdo con McElroy (2002), en cambio, este incluye la infraestructura y cualquier otro tipo de soporte para que el capital humano desarrolle adecuadamente su trabajo. Chen et al. (2004), por otro lado, hacen alusión a la cultura, la estructura, el aprendizaje organi- zativo, los procesos y los sistemas de información, mientras que Joia (2004) estima que está compuesto por la cultura, la estructura, las operaciones, los procesos, las bases de datos, los códigos, los estilos de dirección y las redes internas.

Brooking (1997), por su parte, define dos grandes bloques que estarían incluidos dentro del capital estructural: 1) los activos de infraestructura y 2) los activos de propiedad intelectual. Los primeros están más vinculados con los activos de tipo organizativo (cultura, métodos de dirección, estructura financiera, bases de datos de clientes y sistemas de comunicación), los segundos, con activos de naturaleza tecnológica (knowhow, secretos de fabricación, copyright, patentes y marcas). Finalmente, el Centro de Investigación sobre la Sociedad del Conocimiento (CIC, 2003) desglosa el capital estructural en capital tecnológico y organizativo. El primero contempla aspectos como la investigación, el desarrollo, la dotación tecnológica, la propiedad intelectual y la innovación; el segundo, la cultura, la estructura, el aprendizaje organizativo y los procesos. El conjunto de estas dimensiones, factores o variables propias del capital estructural, así como los autores que los han planteado se muestran de sintética en la siguiente tabla: 
Tabla 2. Dimensiones, factores o variables propias del capital estructural

\begin{tabular}{|c|c|}
\hline Autores & Componentes del capital estructural \\
\hline Bontis (1998) & Rutinas organizativas, estructura, cultura y sistemas de información. \\
\hline Brooking (1997) & $\begin{array}{l}\text { Know-how, secretos de fabricación, patentes, copyright, marcas, cul- } \\
\text { tura, métodos de dirección, estructura financiera, bases de datos y sis- } \\
\text { temas de comunicación. }\end{array}$ \\
\hline $\begin{array}{l}\text { Bueno, Rodríguez \& } \\
\text { Salmador (2003) }\end{array}$ & $\begin{array}{l}\mathrm{I}+\mathrm{D} \text {, dotación tecnológica, propiedad intelectual, resultados de inno- } \\
\text { vación, cultura, estructura, aprendizaje organizativo, procesos y siste- } \\
\text { mas de información. }\end{array}$ \\
\hline Chen et al. (2004) & $\begin{array}{l}\text { Cultura, mecanismos y resultados de innovación, cultura, estructura, } \\
\text { aprendizaje organizativo, procesos y sistemas de información }\end{array}$ \\
\hline CIC (2003) & $\begin{array}{l}\mathrm{I}+\mathrm{D} \text {, dotación tecnológica, propiedad intelectual, resultados de inno- } \\
\text { vación, cultura, estructura, aprendizaje organizativo y procesos. }\end{array}$ \\
\hline Edvinsson \& Malone (1997) & Patentes, marcas, hardware, software, bases de datos y estructura. \\
\hline Euroforum (1998) & Propiedad intelectual, cultura, filosofía, estructura y procesos. \\
\hline $\begin{array}{l}\text { Guthrie, Petty \& } \\
\text { Yongvainich (2004) }\end{array}$ & $\begin{array}{l}\text { Propiedad intelectual, filosofía de dirección, cultura, procesos de di- } \\
\text { rección y sistemas de información. }\end{array}$ \\
\hline Joia (2004) & $\begin{array}{l}\text { Habilidad para crear conocimiento, estructura, procesos operaciones, } \\
\text { cultura, bases de datos, códigos, estilos de dirección y redes internas. }\end{array}$ \\
\hline Kaplan \& Norton (1996) & Procesos internos. \\
\hline McElroy (2002) & Infraestructura y soporte para el capital humano. \\
\hline $\begin{array}{l}\text { Ordóñez de Pablos (2004a, } \\
\text { 2004b) }\end{array}$ & $\begin{array}{l}\text { Resultados de } \mathrm{I}+\mathrm{D} \text {, procesos de ingeniería, innovación, cultura, estruc- } \\
\text { tura, coordinación, rutinas organizativas, sistemas de planificación y } \\
\text { control e infraestructura. }\end{array}$ \\
\hline Saint Onge (1996) & Sistemas, estructura, estrategia y cultura. \\
\hline Sveiby (1997) & $\begin{array}{l}\text { Cultura, organización informal, redes internas y sistemas informáticos } \\
\text { y administrativos. }\end{array}$ \\
\hline
\end{tabular}

Fuente: Elaboración propia.

Determinados los elementos (di- Rodríguez, 2005; Ordóñez de Pamensiones, variables o factores) que blos, 2004a, 2004b; Subramanian \& integran el capital estructural, es Youndt, 2005; Tippins \& Sohi, 2003; necesario concentrarse ahora en su Wilcox, Fowler, \& Zeithaml, 2001; medición. Esta ha sido un objeto de Youndt \& Snell, 2004; Youndt, Suinterés básico para un importante bramanian, \& Snell, 2004). Aunque número de investigadores (Carmeli, no en todos los trabajos se hace un 2004; Carmeli \& Tishler, 2004; CIC, estudio completo de este asunto, sí se 2003; Chen et al., 2004; Gallego \& examinan algunos de los activos in- 
tangibles que lo constituyen. A continuación se presenta una síntesis de las dimensiones del capital estructural consideradas en estos trabajos:

- Cultura: Se entiende a cultura como el conjunto de normas, valores, principios y formas de actuar compartidos por la mayoría de los integrantes de la empresa. Esta es una dimensión presente en la gran mayoría de los trabajos consultados.

- Estructura, sistemas y procesos: Son los modos de organización formal de la empresa que determinan y condicionan la manera de trabajar de sus empleados. Esta es igualmente utilizada de manera amplia.

- Innovación e $I+D$ : Se refiere a los esfuerzos dedicados al diseño, lanzamiento e implementación de nuevos productos, procesos y servicios, tanto para el cliente interno como para el externo. Este factor también es altamente considerado en la literatura consultada.

- Tecnologías de la información y la comunicación: Sobre estas se examina, en particular, el grado de conocimiento y de utilización por parte de los empleados, así como la infraestructura que sobre el particular posee la organización. Esta variable aparece también con bastante frecuencia.

- Modos de conservar el conocimiento: Se encuentran registra- dos en una menor proporción en el interior de la literatura referenciada. Se refieren al soporte, físico o intangible, dispuesto para conservar el conocimiento de la empresa. Este puede estar o no protegido legalmente.

De manera similar a lo que ocurre con el capital humano, en general, no se plantean escalas de medición para las dimensiones descritas. Lo que se hace, por lo general, es que estos aspectos se abordan a través de un conjunto de indicadores.

\section{METODOLOGÍA}

El tipo de investigación realizada corresponde con un estudio empíricoanalítico. Este utilizó como método básico la Case Study Research (Yin, 1994; Bonache, 1999). Contiene entonces rasgos descriptivos, pero analiza, en particular, cómo ocurre el fenómeno organizativo estudiado al interior de su contexto real. El presente es también un trabajo empírico-analítico porque recoge información que, procesada y analizada, permite contrastar los supuestos teóricos que previamente han sido contemplados en la investigación.

El terreno de investigación seleccionado para el presente trabajo fue la Universidad Autónoma de Manizales (UAM), ubicada en una ciudad 
con la que comparte el mismo nombre (Manizales) en Colombia. Esta organización fue elegida, en lo fundamental, por el reconocimiento y prestigio que ha alcanzado en la región del eje cafetero colombiano y por ser esto un posible indicio de que, para lograrlos, ha tenido que explotar, o bien sus activos tangibles, o bien los intangibles o ambos.

Esta organización, como institución de educación superior, ofrece programas académicos (pregrados, especializaciones, maestrías) y propende por transmitir y desarrollar conocimiento en todas sus esferas organizativas. Este terreno resultó ser atractivo, además, por el hecho de que esta universidad no había efectuado antes ningún estudio o medición de sus activos intangibles, razón por la cual llegó a mostrar un gran interés y disposición frente a la propuesta de llevar a cabo la investigación que dio origen al presente artículo.

Para recoger los datos empíricos se utilizó la encuesta denominada 'Identificación y medición del capital intelectual' (Naranjo, Salazar, \& Rubio, 2011) con base en la cual se establecen tres componentes del capital intelectual (humano, estructural y relacional). Esta desglosa cada uno de ellos en diversas dimensiones y establece un conjunto de indicadores a través de los cuáles estas pueden ser medidas.

Tabla 3. Estructura de la encuesta para identificar y medir el capital estructural de la empresa

\begin{tabular}{|l|l|}
\hline \multicolumn{1}{|c}{ Dimensión } & \multicolumn{1}{c|}{ Indicadores } \\
\hline \multirow{5}{*}{ Cultura organizacional } & Conocimiento de la plataforma estratégica (\%) \\
\cline { 2 - 3 } & Misión y visión compartidas (\%) \\
\cline { 2 - 3 } & Orientación a la experimentación y el cambio (\%) \\
\cline { 2 - 3 } & Acciones de bienestar laboral ( $\left.\mathrm{N}^{\circ}\right)$ \\
\cline { 2 - 3 } & Alto grado de compromiso institucional (\%) \\
\cline { 2 - 3 } & Alto nivel de satisfacción laboral (\%) \\
\cline { 2 - 3 } & Participación: ideas y experiencias aportadas (\%) \\
\cline { 2 - 3 } & Canales de comunicación interna (\%) \\
\cline { 2 - 3 } & Inversión en cultura corporativa (\%) \\
\cline { 2 - 3 } & Participación en grupos sociales $(/ \%)$ \\
\hline
\end{tabular}

Continúa 


\begin{tabular}{|c|c|}
\hline Dimensión & Indicadores \\
\hline \multirow{9}{*}{ Tecnología y procesos } & Nivel de utilización de herramientas tecnológicas (\%) \\
\hline & Inversión en tecnología (\%) \\
\hline & Conocimientos almacenados en la intranet $\left(\mathrm{N}^{\mathrm{o}}\right)$ \\
\hline & Nivel de obsolescencia de las bases de datos (\%) \\
\hline & Nivel de obsolescencia de las aplicaciones informáticas (\%) \\
\hline & Nivel de obsolescencia de la maquinaria y el equipo (\%) \\
\hline & Foros virtuales $\left(\mathrm{N}^{\circ}\right)$ \\
\hline & Grado de automatización de los procesos \\
\hline & Calidad de los procesos: certificación (\%) \\
\hline \multirow{8}{*}{$\begin{array}{l}\text { Investigación, desarrollo } \\
\text { e innovación }\end{array}$} & Incentivos por innovar $(\%)$ \\
\hline & Personal en $\mathrm{I}+\mathrm{D}+\mathrm{i}(\%)$ \\
\hline & Inversión en $\mathrm{I}+\mathrm{D}+\mathrm{i}(\%)$ \\
\hline & Nivel de competencia en $\mathrm{I}+\mathrm{D}+\mathrm{i}(\%)$ \\
\hline & Proyectos de $\mathrm{I}+\mathrm{D}+\mathrm{i}$ en ejecución $(\%)$ \\
\hline & Marcas registradas $(\%)$ \\
\hline & Ingresos por nuevos productos $(\%)$ \\
\hline & Ingresos por nuevos servicios $(\%)$ \\
\hline
\end{tabular}

Fuente: Elaboración propia.

Dado que el instrumento aplicado es el mismo utilizado en anteriores investigaciones dentro del macroproyecto de investigación denominado "El capital intelectual en las empresas del eje cafetero", ${ }^{1}$ ya ha sido validado de manera previa y amplia. Este, en efecto, cumple con los criterios de fiabilidad exigidos para dicho tipo de estudios (revisión exhaustiva de la literatura especializa- da, juicio de expertos, prueba piloto, alfa de Cronbach y correlaciones de Pearson).

Respondiendo a la tendencia encontrada en diversos modelos de capital intelectual consultados, así como en los estudios efectuados por autores de renombre para su medición, no se contemplaron escalas de medida, sino indicadores que, a través

1 Macroproyecto de investigación desarrollado en el contexto de la Maestría en Administración de Negocios de la Universidad Autónoma de Manizales. Mediante este, desde el 2010, se ha medido el capital intelectual a un total de catorce empresas, cada una como un estudio independiente. 
de datos duros, permitieran establecer el estado, en cada uno de los periodos considerados, de las dimensiones incluidas. El hecho de contemplar diferentes períodos obedece a la concepción dinámica del capital intelectual, según la cual resulta imperioso mirar el flujo de los activos que lo componen, de manera que se puedan establecer las variaciones producidas e interpretarlas. Ello evitando la lectura estática, que resulta bastante limitada e inexacta para comprender el comportamiento de dicho capital. En este caso se efectuó la medición para los años 2011 y 2012.

Antes de proceder a diligenciar la encuesta se envió una carta al director académico solicitando su visto bueno para realizar el estudio en la UAM. Una vez se obtuvo la aprobación, se realizó una visita al director administrativo y financiero, ello con el fin de darle a conocer los objetivos del estudio, los alcances y la confidencialidad en el manejo de la información. Con el aval de la Dirección de la Universidad la encuesta se socializó y se aplicó a todas las personas que debían participar en el proceso. Los datos requeridos por la encuesta fueron provistos por la organización, contando con la asesoría y acompañamiento permanente de los investigadores $\mathrm{y}$, cuando la ocasión lo hizo necesario, con su participación directa en el recaudo de la información o el cálculo del indicador.

También se extrajo información de los informes que cada una de las direcciones (Académica, Administrativa y Financiera, Desarrollo Humano y Secretaría General) presentan a la Asamblea General de Corporados. El hecho de considerar este conjunto de acciones para la aplicación de la encuesta permitió el contacto, la implicación y el aporte de todas las personas requeridas para obtener los datos demandados por el estudio.

Para el procesamiento de la información se creó una base de datos en Microsoft Excel ${ }^{\circledR}$ y se llevaron a cabo reuniones adicionales con los directivos y los responsables de los procesos o áreas pertinentes. Ello con el fin de compartir información que contribuyera a explicar el comportamiento de las cifras encontradas, la cual, posteriormente, fue utilizada en el análisis del capital estructural de la Universidad.

\section{RESULTADOS}

En la dimensión del capital estructural se incluyen todos aquellos elementos que componen la organización interna y se llevan a la práctica en la entidad con el fin de desempeñar sus funciones de manera óptima. 
Entre estos se pueden señalar las bases de datos, los cuadros de organización, los manuales de procesos, la propiedad individual (patentes, marcas o cualquier elemento intangible que pueda ser protegido de acuerdo con la propiedad intelectual) y todos aquellos otros aspectos cuyo valor para la empresa sea superior al material (Roman, 2005).

La encuesta utilizada como base para identificar el capital estructural en la UAM cuenta con tres dimensiones (cultura organizacional, tecnología y procesos e investigación desarrollo e innovación) que son medidas a través de veintisiete indicadores (ver tabla 3). Lo obtenido por el trabajo, en relación con estas, se presenta a continuación:

\subsection{Cultura organizacional}

La cultura organizacional puede facilitar el desarrollo de una estrategia que conduzca a la empresa a la consecución de sus objetivos o, por el contrario, puede impedir su cumplimiento o generar retrasos en el crecimiento de la compañía. Por este motivo se considera que este factor influye directamente sobre los resultados de la actividad de cada organización. Los resultados obtenidos en relación con esta dimensión se presentan en la siguiente tabla:

Tabla 4. Cultura organizacional

\begin{tabular}{|l|c|c|}
\hline \multicolumn{1}{|c|}{ Indicador } & 2011 & 2012 \\
\hline Conocimiento de la plataforma estratégica (\%) & $82,09 *$ & $84,84^{*}$ \\
\hline Misión y visión compartidas (\%) & $82,09 *$ & $84,84^{*}$ \\
\hline Orientación a la experimentación y el cambio (\%) & No disponible & No disponible \\
\hline Número de acciones de bienestar laboral & 120 & 125 \\
\hline Alto grado de compromiso institucional (\%) & No disponible & No disponible \\
\hline Alto nivel de satisfacción laboral (\%) & No disponible & No disponible \\
\hline Participación: ideas y experiencias aportadas (\%) & No disponible & No disponible \\
\hline Número de canales de comunicación interna (n $\left.{ }^{\circ}\right)$ & 7 & 7 \\
\hline Inversión en cultura corporativa (\%) & 8,42 & 9,07 \\
\hline Empleados que participan en grupos sociales (\%) & 31,30 & 30,87 \\
\hline
\end{tabular}

* Valor estimado

Fuente: Elaboración propia. 
En el año 2006 la UAM realizó un ejercicio de redireccionamiento estratégico. En este se revisaron la misión y los valores institucionales, se modificó una parte de la visión y se formularon nuevos objetivos estratégicos. Estos últimos fueron revisados en 2012, como parte de los procesos de mejoramiento continuo, en particular, con el fin de dar respuesta a las nuevas demandas de la sociedad.

Las estrategias que ha implementado la UAM para el logro de sus objetivos se han enfocado en temas como la pertenencia, la innovación, la eficiencia administrativa, el bienestar, la proyección nacional e internacional, el impacto y la presencia en el entorno.

Conviene mencionar que toda la información referente a la cultura organizativa de la UAM (misión, visión, valores y objetivos estratégicos) se encuentra publicada en la página web institucional y está disponible para el acceso a ella por parte del público en general, lo que facilita la consulta de la misma por parte de todos los interesados. Estos aspectos son socializados con los empleados a través de una capacitación virtual que fue desarrollada con este fin por parte de la Dirección de Desarrollo Humano.

En cuanto al bienestar laboral, para la Universidad es importante que sus empleados tengan un alto sentido de pertenencia y que se encuentren satisfechos. Por ello es frecuente que se ofrezcan programas en el ámbito social como la realización de múltiples actividades artísticas, deportivas y culturales, entre otras que permiten un crecimiento personal al empleado. Con este fin se han ido fortaleciendo también diversos espacios adicionales, como el gimnasio, el salón de música, la cancha de microfútbol y otros. Estos permiten la realización de las mencionadas actividades de una manera más grata. Sin embargo, se considera importante buscar nuevas estrategias en esta materia para que un mayor número de empleados se vincule a las actividades ofrecidas por la Universidad.

Por otro lado, aunque en la UAM no se restringe la opinión de los empleados acerca de posibles mejoras e ideas en relación con eventuales nuevos servicios, no existe una oficina $u$ otro espacio especializado que permita centralizar estos aportes. Como resultado, esta información no se puede cuantificar ni es posible generar un indicador que permita medirla. En relación con el indicador de canales de comunicación interna, se identifica que la UAM cuenta con página web, correo electrónico, flash informativo, reporte informativo noticioso, carteleras, medios telefónicos y pantallas informativas. A través de estos 
mecanismos mantiene una comunicación de doble vía con sus clientes internos.

Finalmente, es importante mencionar que durante la presente investigación se pudo observar que este factor es uno de los que más trabajo requiere a futuro. Ello dado que de los diez indicadores estudiados en relación con el mismo, cuatro no están disponibles y dos debieron ser estimados por parte del equipo investigador.

\subsection{Tecnología y procesos}

Como se indicó anteriormente, el capital humano es considerado el activo más valioso de una organización; sin embargo, por sí solo no representa una ventaja competitiva para la misma, ya que es necesario que las personas cuenten con la infraestructura adecuada para poder llegar a ser más efectivas en su trabajo.
En los últimos años el uso de la tecnología ha venido tomando fuerza. Este representa un factor determinante en la agilidad de los procesos y posterior consecución de óptimos resultados, lo que proporciona una ventaja competitiva a las empresas que hacen un uso adecuado de la misma.

Desde el año 2005 la UAM ha venido fortaleciendo su infraestructura tecnológica (hardware, software y redes), pues la institución es consciente de la importancia que esta ha tomado en los últimos años y del destacado lugar que ella ocupa para mejorar y agilizar los procesos que se realizan a diario. Como resultado de lo anterior se observa un incremento gradual en la inversión en esta materia.

A continuación se presentan los datos obtenidos para los años 2011 y 2012 en lo relativo al uso de la tecnología en la UAM (ver tabla 5).

Tabla 5. Tecnología y procesos

\begin{tabular}{|l|l|l|}
\hline \multicolumn{1}{|c|}{ Indicador } & \multicolumn{1}{|c|}{2011} & \multicolumn{1}{c|}{2012} \\
\hline Nivel de utilización de herramientas tecnológicas (\%) & $77,78 \%$ & $88,89 \%$ \\
\hline Inversión en tecnología (\%) & $1,71 \%$ & $1,81 \%$ \\
\hline Número de conocimientos almacenados en la intranet & 31 & 388 \\
\hline Grado de automatización de procesos & No disponible & No disponible \\
\hline Calidad de los procesos & No disponible & No disponible \\
\hline
\end{tabular}




\begin{tabular}{|l|l|l|}
\hline \multicolumn{1}{|c|}{ Indicador } & \multicolumn{1}{|c|}{2011} & \multicolumn{1}{|c|}{2012} \\
\hline Nivel de obsolescencia de las bases de datos (\%) & $10 *$ & $10 *$ \\
\hline Nivel de obsolescencia de las aplicaciones informáticas (\%) & 0 & 0 \\
\hline Nivel de obsolescencia de la maquinaria y equipo (\%) & 5 & 4 \\
\hline Número de foros virtuales & 3 & 35 \\
\hline
\end{tabular}

* Valor estimado

Fuente: Elaboración propia.

La utilización de herramientas tecnológicas tuvo un incremento anual del $11,11 \%$. Esto es muy positivo para la Universidad, ya que facilita la evolución de la organización hacia la gestión por procesos, genera confiabilidad y efectividad en el análisis de la información y agilidad en la obtención de los resultados. La inversión anual en materia de tecnología en los años 2011 y 2012 por parte de la Universidad alcanzó los \$429328000 y \$523082000, respectivamente. Esto le ha permitido actualizar continuamente su plataforma tecnológica.

Los indicadores de automatización tecnológica y de calidad no están disponibles, ya que actualmente la Universidad se encuentra en proceso de cambio de gestión por áreas a gestión por procesos. Esto dificulta estimar un porcentaje de automatización global. Sin embargo, se constata la existencia de apoyo tecnológico en varias áreas y procesos, entre los que se destacan, además de otros, conta- bilidad, registro académico, mercadeo y servicio al cliente, nómina y biblioteca.

El nivel de obsolescencia de las bases de datos es del $10 \%$, puesto que aún existen algunas falencias en ciertas áreas como la de graduados, que no cuenta aún con la suficiente actualización. El nivel de obsolescencia de las aplicaciones informáticas, por su parte, es del $0 \%$ y el de la maquinaria y equipos del $4,5 \%$. Esto debido a que se tienen contratos de actualización y mantenimiento anuales, tanto para motores de bases de datos como para las diferentes aplicaciones que apoyan las funciones de todos los empleados de la Universidad. Dichos contratos permiten que cada vez que es lanzada al público una nueva versión de estos recursos la UAM tenga derecho a instalarla y a usarla sin mayores demoras.

El indicador de conocimientos almacenados en la intranet tuvo un in- 
cremento anual de 357 documentos. Esto debido a que en el año 2011 estaban almacenados textos institucionales que debían ser de conocimiento para la comunidad en general, como reglamentos, políticas y estatutos, entre otros. A partir del año 2012, además de estos, se realiza la publicación de los documentos en los que se recopilan los procesos institucionales, trabajados ahora en gestión por procesos. Todos estos escritos son actualizados año tras año con el fin de tener información veraz y oportuna para todos los stakeholders de la institución.

Finalmente, se encuentra que la UAM ha hecho esfuerzos importantes en esta dimensión, gracias a los cuales, entre otras acciones, se ha incrementado el uso de la plataforma virtual. Esta, inicialmente, era utilizada solo por los estudiantes en sus cursos, pero hoy hacen uso de ella también los empleados. En un principio se tenían además pocos foros de participación (3), sin embargo, en 2012 se habilitó una serie de ayudas para los trabajadores que tengan el interés de mejorar sus habilidades. Entre estas se destacan foros como "Las TIC en la productividad personal", "Ambientes b-learning con Moodle", "Aprendizaje colaborativo con Web
2.0", "Objetos de aprendizaje" y "Ofimática para empleados administrativos", entre otros.

\subsection{Investigación, desarrollo e innovación}

Para una institución de educación superior, por su naturaleza, este es uno de los factores claves a trabajar, pues hace parte de sus funciones sustantivas. En efecto, entre otras actividades básicas, es a través del desempeño en esta materia que los diversos entes externos evalúan si una universidad cuenta con una alta calidad o no. Por esta razón, la UAM, al igual que las demás universidades regionales, nacionales e internacionales, cuenta con una unidad dedicada exclusivamente a gestionar los procesos de investigación de la institución.

Es importante mencionar que este factor fue analizado únicamente para el área administrativa de la Universidad, ya que en la actualidad se realiza otro proceso de investigación dedicado exclusivamente a revisar el capital intelectual, de forma específica, en función de la parte investigativa de la institución. Los resultados obtenidos en esta materia, considerando lo que se acaba de señalar, se presentan en la siguiente tabla: 
Tabla 6. Investigación, desarrollo e innovación

\begin{tabular}{|l|l|l|}
\hline Indicador & 2011 & 2012 \\
\hline Incentivos por innovar (\%) & 0 & 0 \\
\hline Personal en I+D+i (\%) & 16,33 & 14,28 \\
\hline Inversión en I+D+i (\%) & 7,2 & 7,2 \\
\hline Nivel de competencia en I+D+i (\%) & No disponible & No disponible \\
\hline Proyectos de I+D+i en ejecución (\%) & 0 & 0 \\
\hline Número de marcas registradas & 0 & 1 \\
\hline Ingresos por nuevos productos (\%) & N/A & N/A \\
\hline Ingresos por nuevos servicios (\%) & 0,8 & 1,48 \\
\hline
\end{tabular}

Fuente: Elaboración propia.

Se encuentra que en el área administrativa el porcentaje de investigación es muy bajo. Esto debido, por supuesto, a que la misma no es una competencia característica de esta área, pero también a que la Universidad, como se indicó antes, cuenta con su propia unidad dedicada específicamente a la labor de investigación.

La inversión en investigación es del $7,2 \%$. Esto permite el cumplimiento de dos objetivos estratégicos importantes, a saber:

- Potenciar la investigación y la innovación de la UAM en los ámbitos regional, nacional e internacional, con el fin de contribuir al desarrollo sostenible y al bienestar de las poblaciones, en un marco localmente pertinente e internacionalmente competitivo.

- Contribuir a la consolidación de una cultura institucional basada en la generación, apropiación y difusión del conocimiento científico, la innovación y el aprendizaje constante en la comunidad académica, con el fin de hacer de la investigación un hábito permanente.

Vale la pena mencionar que en 2012 la institución adquirió su primera patente, producto del trabajo de uno de sus grupos de investigación. Esto tiene un gran valor para la Universidad y la ciudad, ya que hasta el momento ninguna otra universidad privada de la región había alcanzado algo así.

\section{CONCLUSIONES}

Finalizado el análisis de los aspectos que conforman el capital estructural de la UAM se puede concluir que, si bien esta organización tiene una gran fortaleza en todo lo que se 
refiere a infraestructura tecnológica y proceso de $\mathrm{I}+\mathrm{D}+\mathrm{i}$, es necesario trabajar en la cultura organizacional pues, como se evidenció, existen múltiples indicadores que no se evalúan y que podrían ser de gran ayuda para la toma de decisiones institucionales. Por medio de este estudio se pueden detectar problemas internos de la Universidad y plantear soluciones que permitan resolverlos rápidamente, así como integrar a los empleados con los objetivos buscados por esta y conocer las necesidades del personal. Esto para buscar estrategias por medio de las cuales ellas puedan ser satisfechas $y$, de esa manera, mejorar su motivación.

En términos generales, la UAM puede responder oportunamente a las necesidades de sus clientes $y$, en general, de la sociedad. Cuenta en la actualidad con los recursos humanos y técnicos necesarios para generar una ventaja competitiva. Además, ha trabajado arduamente para lograr que sus empleados se sientan satisfechos en la institución.

Por último, se recomienda realizar mediciones a la efectividad de los diferentes canales de comunicación. Estos constituyen un excelente medio para obtener información directa desde las fuentes y para poder así plantear soluciones o estrategias que impacten la organización.
Finalmente, se recomienda también realizar estudios de clima organizacional anuales, ya que hasta el momento, según la información obtenida a través de la Unidad de Gestión Humana, los estudios se hacen a través de la administradora de riesgos laborales (ARL) y, de hecho, el último fue en 2010. En este trabajo se pudo evidenciar que el $70 \%$ de los empleados se encuentra comprometido y satisfecho con la Universidad.

\section{REFERENCIAS}

Bonache, J. (1999). El estudio de casos como estrategia de construcción teórica: características, críticas y defensas. Cuadernos de Economía y Dirección de la Empresa, 3, 123-140.

Bontis, N. (1996). There's a price on your head: Managing intellectual capital strategically. $B u$ siness Quarterly, 60(4), 41-47.

Bontis, N. (1998). Intellectual capital: An exploratory study that develops measures and models. $\mathrm{Ma}$ nagement Decision, 36(2), 63-76. Brooking, A. (1997). El capital intelectual: el principal activo de las empresas del tercer milenio. Barcelona: Paidós Ibérica.

Bueno Campos, E. (1998). El capital intangible como clave estratégica en la competencia actual. Boletín de Estudios Económicos, 53(164), 207- 229. 
Bueno, E., Rodríguez, O., \& Salmador, M. P. (2003). La importancia del capital social en la sociedad del conocimiento: propuesta de un modelo integrador de capital intelectual (Ponencia presentada al Primer Congreso Internacional y Virtual de Intangibles, Madrid, España).

Cañibano, L., \& Sánchez, M. P. (2004). Medición, gestión e información de intangibles: lo más nuevo. Contabilidad y dirección. Medición, control y gestión de los intangibles. Barcelona, ACCID - DEUSTO.

Carmeli,A. (2004). The link between organizational elements, perceived external prestige and performance. CorporateReputation Review, 6, 314-331.

Carmeli, A., \& Tishler, A. (2004). The relationships between intangible organizational elements and organizational performance. Strategic Management Journal, 25(13), 1257-1278.

Centro de Investigación sobre la Sociedad del Conocimiento (CIC) (2003). Modelo intellectus: medición y gestión del capital intelectual. Madrid: Universidad Autónoma de Madrid.

Chen, J., Zhu, Z., \& Yuan, H. (2004). Measuring intellectual capital: A new model and empirical study. Journal of Intellectual Capital, 5(1), 195-212.
Edvinsson, L., \& Malone, M. (1997). Intellectual capital: Realizing your company's true value by finding its hidden brainpower. Nueva York: Harper Collins.

Euroforum Escorial (1998). Medición del capital intelectual: modelo intellect, I.U. Madrid: Euroforum Escorial.

Gallego, I., \& Rodríguez, L. (2005). Situation on intangibles assets in Spanish firms: An empirical analysis. Journal of Intellectual Capital, 6(1), 105-126.

Guthrie, J., Petty, R., \& Yongvainich, K. (2004). Using content analysis as a research method to inquire into intellectual capital reporting. Journal of Intellectual Capital. 5(2), p. 282-293.

Joia, A. (2004). Are frequent customer always a company's intangible asset? Some findings drawn from an exploratory case study. Journal of Intellectual capital, 5(4), 586-601.

Kaplan, R. S., \& Norton, D. P. (1996). The balanced scorecard: Translating strategy into action. Boston: Harvard Business School Press.

Klein, D. (1998). A gestao estratégica do capital intelectual. Río de Janeiro: Qualitymark.

Low, J., \& Cohen, P. (2004). La ventaja invisible. Cómo impulsan los intangibles el rendimiento empresarial. Barcelona: Urano. 
Mantilla, S. A. (2004). Contabilidad intelectual \& contabilidad del conocimiento. Bogotá: Ecoe.

Marr, B., Gupta, O., Pike, S., \& Roos, G. (2003). Intellectual capital and knowledge management effectiveness. Management Decision - London The Bradford. (41) 8, pp. 5-24.

McElroy, M. W. (2002). Social innovation capital. Journal of Intellectual Capital, 3(1), 30-39.

Mouritsen, J., Bukh, P. N., Larsen, H. T., \& Johansen, M. R. (2002). Developing and managing knowledge through intellectual capital statements. Journal of Intellectual Capital, 3(1), 10-29.

Naranjo, C., Salazar, L., \& Rubio, J. (2011). El capital intelectual en Bellota - Colombia S.A. (Tesis de maestría. Universidad Autónoma de Manizales, Manizales, Colombia).

Nazari, J., \& Herremans, M. (2007). Extended VAIC model: Measuring intellectual capital components. Journal of Intellectual Capital, 8(4), 595-609.

Ordóñez de Pablos, P. (2004a). Las cuentas de capital intelectual como complemento del informe anual. Economía Industrial, 357, 63-74.

Ordóñez de Pablos, P. (2004b). Measuring and reporting structural capital: Lessons from European learning firms. Journal of Intellectual Capital, 5(4), 629-647.
Rastogi, P. N. (2002). Knowledge management and intellectual capital as a paradigm of value creation. Human System Management, 21(3), 229-240.

Roman, N. (2005). Capital intelectual generador de éxito en las empresas.VisiónGerencial,3(2), 67-79.

Saint-Onge, H. (1996). Tacit knowledge: The key to strategic alignmentofintellectualcapital.Strategy and Leadership, 24(2), 10-14. Steward, T. (1998). La nueva riqueza de las organizaciones: el capital intelectual. Buenos Aires: Granica.

Subramanian, M., \& Youndt, M. (2005). The influence of intellectual capital on the types of innovative capabilities. Academy of Management Journal, 48(3), 450463.

Sveiby, K. E. (1997). The new organizational wealth: Managing and measuring knowledge-based assets. San Francisco: BerretKoehler.

Swart, J. (2006). Intellectual capital: Disentangling an enigmatic concept. Journal of Intellectual Capital, 7(2), 136-159.

Tippins, M., \& Sohi, R. (2003). Iт competency and firm performance: Is organizational learning a missing link? Strategic Management Journal, 24(8), 745-761.

Wilcox, A., Fowler, S., \& Zeithaml, C. (2001). Managing organiza- 
tional competencies for competitive advantage: The middle-management edge. Academy of $\mathrm{Ma}$ nagement Perspectives, 15(2), 95-106.

Yin, R. (1994).Case study research. Design and methods. Newbury Park - Londres: SAGE.

Youndt, M., \& Snell, S. (2004). Human resource configurations, in- tellectual capital and organizational performance. Journal of Managerial Issues. 16(3), 337360.

Youndt,M.,Subramaian,M.,\&Snell, S. (2004). Intellectual capital profiles: An examination of investments and returns. Journal of Management Studies, 41(2), 335361. 